\title{
Prescribing Patterns for Treatment of Mycobacterium avium Complex and M. xenopi Pulmonary Disease in Ontario, Canada, 2001-2013
}

\author{
Sarah K. Brode, Hannah Chung, Michael A. Campitelli, Jeffrey C. Kwong, Alex Marchand-Austin, \\ Kevin L. Winthrop, Frances B. Jamieson, Theodore K. Marras
}

\section{Medscape ACTIVITY}

In support of improving patient care, this activity has been planned and implemented by Medscape, LLC and Emerging Infectious Diseases. Medscape, LLC is jointly accredited by the Accreditation Council for Continuing Medical Education (ACCME), the Accreditation Council for Pharmacy Education (ACPE), and the American Nurses Credentialing Center (ANCC), to provide continuing education for the healthcare team.

Medscape, LLC designates this Journal-based CME activity for a maximum of 1.00 AMA PRA Category 1 Credit(s) ${ }^{\mathrm{TM}}$.

Physicians should claim only the credit commensurate with the extent of their participation in the activity.

Successful completion of this CME activity, which includes participation in the evaluation component, enables the participant to earn up to $1.0 \mathrm{MOC}$ points in the American Board of Internal Medicine's (ABIM) Maintenance of Certification (MOC) program. Participants will earn MOC points equivalent to the amount of CME credits claimed for the activity. It is the CME activity provider's responsibility to submit participant completion information to ACCME for the purpose of granting ABIM MOC credit.

All other clinicians completing this activity will be issued a certificate of participation. To participate in this journal CME activity: (1) review the learning objectives and author disclosures; (2) study the education content; (3) take the post-test with a $75 \%$ minimum passing score and complete the evaluation at http://www.medscape.org/journal/eid; and (4) view/print certificate. For CME questions, see page 1442.

Release date: June 14, 2019; Expiration date: June 14, 2020

Learning Objectives

Upon completion of this activity, participants will be able to:

- Identify the percentage of older adults with MAC-PD and Mx-PD in Ontario, Canada, who received antimicrobial therapy and factors associated with receipt of treatment, according to a retrospective cohort study

- Determine the percentage of older adults with MAC-PD and Mx-PD in Ontario, Canada, who received guidelinerecommended treatment and compare this percentage with that in other countries, according to a retrospective cohort study

- Ascertain the percentage of older adults with MAC-PD and Mx-PD in Ontario, Canada, who received treatment that could lead to macrolide resistance and factors associated with receipt of such treatment, according to a retrospective cohort study

CME Editor

P. Lynne Stockton Taylor, VMD, MS, ELS(D), Technical Writer/Editor, Emerging Infectious Diseases. Disclosure: P. Lynne Stockton Taylor, VMD, MS, ELS(D), has disclosed no relevant financial relationships.

CME Author

Laurie Barclay, MD, freelance writer and reviewer, Medscape, LLC. Disclosure: Laurie Barclay, MD, has disclosed no relevant financial relationships.

Authors

Disclosures: Sarah Brode, MD, MPH; Hannah Chung, MPH; Michael Campitelli, MPH; Jeff Kwong, MD; Alex Marchand-Austin, MD, MSc; and Frances B. Jamieson, MD, have disclosed no relevant financial relationships. Kevin Winthrop, MD, MPH, has disclosed the following relevant financial relationships: served as an advisor or consultant for Insmed Incorporated, Johnson \& Johnson Pharmaceutical Research \& Development L.L.C., Paratek Pharmaceuticals, RedHill Biopharma Inc.; received grants for clinical research from Insmed Incorporated. Theodore Marras, MD, has disclosed the following relevant financial relationships: served as an advisor or consultant for Insmed Incorporated, RedHill Biopharma Inc.; served as a speaker or a member of a speakers bureau for AstraZeneca Pharmaceuticals LP, Novartis Pharmaceuticals Corporation; received grants for clinical research from Insmed Incorporated.

Author affiliations: West Park Healthcare Centre, Toronto, Ontario, Canada (S.K. Brode); ICES, Toronto (S.K. Brode, H. Chung, M.A. Campitelli, J.C. Kwong); University of Toronto, Toronto (S.K. Brode, J.C. Kwong, F.B. Jamieson, T.K. Marras); University Health Network and Sinai Health System, Toronto (S.K. Brode, T.K. Marras); Toronto Western Family Health
Team, Toronto (J.C. Kwong); Public Health Ontario, Toronto (J.C. Kwong, A. Marchand-Austin, F.B. Jamieson); Oregon Health and Science University, Portland, Oregon, USA (K.L. Winthrop)

DOI: https://doi.org/10.3201/eid2507.181817 
Surveys suggest that clinicians diverge from guidelines when treating Mycobacterium avium complex (MAC) pulmonary disease (PD). To determine prescribing patterns, we conducted a cohort study of adults $\geq 66$ years of age in Ontario, Canada, with MAC or Mycobacterium xenopi PD during 2001-2013. Using linked laboratory and health administrative databases, we studied the first treatment episode ( $\geq 60$ continuous days of $\geq 1$ of a macrolide, ethambutol, rifamycin, fluoroquinolone, linezolid, inhaled amikacin, or, for M. xenopi, isoniazid). Treatment was prescribed for $24 \%$ MAC and $15 \%$ of $M$. xenopi PD patients. Most commonly prescribed was the recommended combination of macrolide, ethambutol, and rifamycin, for $47 \%$ of MAC and $36 \%$ of M. xenopi PD patients. Among MAC PD patients, $20 \%$ received macrolide monotherapy and $33 \%$ received regimens associated with emergent macrolide resistance. Although the most commonly prescribed regimen was guidelines-recommended, many regimens prescribed for MAC PD were associated with emergent macrolide resistance.

$\mathrm{N}$ ontuberculous mycobacteria (NTM) pulmonary disease (PD) is increasing in North America (1-3). The 2 most common causes of NTM PD in Ontario, Canada, are Mycobacterium avium complex (MAC) and M. xenopi (1). Treatment guidelines detailing evidence-based treatment regimens for MAC PD have been published; the first-line recommendation is a 3-drug combination of a macrolide, ethambutol, and a rifamycin (hereafter referred to as standard triple therapy) (4). Although there are no evidence-based treatment regimens for $M$. xenopi PD, expertsupported regimens have been suggested (4). Physician surveys suggest that, when treating MAC PD, clinicians frequently diverge from guideline recommendations $(5,6)$. However, population-based data on treatment practices for MAC PD or M. xenopi PD are lacking. Our study objective was to examine antimicrobial drug prescribing patterns for MAC PD or M. xenopi PD in older Ontario residents.

\section{Methods}

Our retrospective cohort study used population-based linked laboratory and health administrative databases in Ontario, Canada, described previously (7). These datasets were linked by using unique encoded identifiers and analyzed at ICES (Toronto, Ontario, Canada). Ontario is Canada's most populous province; the population in 2013 was 13.5 million residents. Ontario has a single-payer healthcare system that provides universal access to medically necessary inpatient and outpatient services and prescription drugs to adults $\geq 65$ years of age. Ontario also has a reference mycobacteriology laboratory that processes $>95 \%$ of NTM specimens for the province (8).

Our study cohort consisted of adults $\geq 66$ years of age with incident MAC PD or M. xenopi PD, defined according to American Thoracic Society/Infectious Diseases Society of America (ATS/IDSA) microbiological criteria (4), during 2001-2013; observations ended December 31, 2014. The date of diagnosis was defined as the date of collection of the first positive culture sample. To avoid confusion regarding the species for which the treatment was intended, we excluded patients who met ATS/IDSA microbiological criteria for infection with $>1$ NTM species during followup. We also excluded patients who died within 1 year of NTM PD diagnosis (and in a sensitivity analysis those who died within 2 years of diagnosis) and patients who had culture-confirmed tuberculosis (TB) after NTM PD diagnosis. We looked back 3 years before the study period to find preexisting isolation of NTM and M. tuberculosis complex; we excluded patients for whom NTM had been isolated during the 3-year look-back period and M. tuberculosis complex within 18 months of NTM PD diagnosis.

We studied the first treatment episode after NTM PD diagnosis, defined as $\geq 60$ continuous days of treatment (either daily or intermittent) with $\geq 1$ drug or class commonly used to treat MAC PD or M. xenopi PD (macrolide, ethambutol, rifamycin, fluoroquinolone, linezolid, inhaled amikacin, or, for M. xenopi PD, isoniazid), started within 1 year of any culture positive for the causative NTM species/complex and ended at the time of a $\geq 60$ day treatment interruption. To allow for patients who refilled their prescriptions late, we defined treatment as continuous if they filled their next prescription for the same antimicrobial drug class within 1.5 times the number of days supplied in their last prescription. We also examined antimicrobial drug treatment given in the first 18 months after the start of the first treatment episode (i.e., not ending at a treatment interruption of $\geq 60$ days) to capture breaks in therapy and switches between regimens (each defined as lasting $\geq 60$ days).

We collected data about patient demographics and underlying conditions at the time of NTM PD diagnosis, prescribing physician specialty, treatment details, and medication use and healthcare use associated with asthma and chronic obstructive pulmonary disease (COPD) at the time of NTM PD diagnosis (Appendix, https://wwwnc.cdc.gov/ EID/article/25/7/18-1817-App1.pdf) (9-16). To compare patient characteristics, we used analysis of variance for continuous variables and $\chi^{2}$ tests for categorical variables. To determine patient characteristics associated with initial prescription of macrolide monotherapy for $\geq 60$ continuous days versus other regimens, we also performed bivariate and multivariable logistic regression analyses among MAC PD patients; included variables were selected a priori on the basis of clinical relevance. We used SAS version 9.4 (SAS Institute, https://www.sas.com) for all analyses and considered a 2 -sided $p$ value of $\leq 0.05$ to be significant. This study was approved by research ethics boards at University Health Network and Public Health Ontario. 


\section{Results}

Of the 3,163 patients with MAC PD and 1,048 with $M$. xenopi PD, we excluded 329 (10.4\%) MAC PD and 120 (11.4\%) M. xenopi PD patients because they also met microbiological criteria for infection with another species of NTM PD or had TB. Treatment was received by 688 $(24.2 \%)$ of the 2,834 patients with exclusively MAC PD and $142(15.3 \%)$ of the 928 with exclusively M. xenopi PD. A sensitivity analysis limited to patients who survived $\geq 2$ years after NTM PD diagnosis indicated that treatment was received by $622 / 2533(24.6 \%)$ of MAC PD patients and $114 / 785$ (14.5\%) of M. xenopi PD patients. Compared with MAC PD patients who did not receive treatment, those who did receive treatment were younger (mean age 75.6 vs. 76.9 years); more likely to be female (59.4 vs. $54.8 \%$ ); more likely to reside in neighborhoods in the higher income quintile and rural settings; more likely to have bronchiectasis, COPD, and interstitial lung disease; and less likely to have diabetes mellitus, chronic kidney disease, and lung cancer (Table 1). Compared with M. xenopi PD patients who did not receive treatment, those who did receive treatment were more likely to have COPD (83.1 vs 63.0\%) (Table 1).

The median time from NTM PD diagnosis to start of the first treatment episode was 77 (interquartile range [IQR] 28-239) days for MAC PD and 79 (IQR 40-199) days for $M$. xenopi PD patients. Among MAC PD patients who received treatment, the most commonly prescribed drug in the first treatment episode was a macrolide $(87.1 \%)$, followed by ethambutol $(70.2 \%)$, a rifamycin $(58.6 \%)$, and a fluoroquinolone (33.7\%) (Table 2). These drugs were prescribed with similar frequency for $M$. xenopi PD patients. No linezolid was prescribed. Isoniazid, assessed for M. xenopi PD disease only, was rarely prescribed $(\leq 5[\leq 3.5 \%]$ patients). Amikacin, as recorded in our databases, was dispensed for inhalation and was rarely used; for $\leq 5(\leq 0.7 \%)$ MAC PD and 0 M. xenopi PD patients, inhaled amikacin for $\geq 60$ days was prescribed.

Table 1. Baseline characteristics of patients who did and did not receive treatment for MAC PD and M. xenopi PD, Ontario, Canada, 2001-2013*

\begin{tabular}{|c|c|c|c|c|c|c|}
\hline \multirow[b]{2}{*}{ Characteristic } & \multicolumn{3}{|c|}{ MAC PD } & \multicolumn{3}{|c|}{ M. xenopi PD } \\
\hline & Treated, $\mathrm{n}=688 \dagger$ & Untreated, $\mathrm{n}=2,146$ & $p$ value & Treated, $\mathrm{n}=142 \dagger$ & Untreated, $\mathrm{n}=786$ & $p$ value \\
\hline Sex & & & 0.031 & & & 0.248 \\
\hline $\mathrm{F}$ & $409(59.4)$ & $1,175(54.8)$ & & $61(43.0)$ & $380(48.1)$ & \\
\hline $\mathrm{M}$ & $279(40.6)$ & $971(45.2)$ & & $81(57.0)$ & $407(51.8)$ & \\
\hline Age, mean \pm SD & $75.6 \pm 5.94$ & $76.9 \pm 6.65$ & $<0.001$ & $75.1 \pm 5.92$ & $76.1 \pm 6.50$ & 0.077 \\
\hline Income quintile & & & 0.018 & & & 0.932 \\
\hline 1 (lowest) & $142(20.6)$ & $550(25.6)$ & & $32(22.5)$ & $164(20.9)$ & \\
\hline 2 & $146(21.2)$ & $428(19.9)$ & & $33(23.2)$ & $178(22.6)$ & \\
\hline 3 & $124(18.0)$ & $425(19.8)$ & & $26(18.3)$ & $131(16.7)$ & \\
\hline 4 & $117(17.0)$ & $354(16.5)$ & & $24(16.9)$ & $157(20.0)$ & \\
\hline 5 (highest) & $\begin{array}{c}155-159 \\
(22.5-23.1)\end{array}$ & $382(17.8)$ & & $\begin{array}{c}22-27 \\
(155-1901)\end{array}$ & $\begin{array}{c}153-158 \\
(195-201)\end{array}$ & \\
\hline Missing & $\leq 5(\leq 0.7)$ & $7(0.3)$ & & $\leq 5(\leq 3.5)$ & $\leq 5(\leq 0.7)$ & \\
\hline Residency & & & $<0.001$ & & & 0.288 \\
\hline Rural & $37(5.4)$ & $53(2.5)$ & & $\leq 5(\leq 3.5)$ & $8(1.0)$ & \\
\hline Suburban & 89 (12.9) & $159(7.4)$ & & $9-14(6.3-9.9)$ & $38(4.8)$ & \\
\hline Urban & $562(81.7)$ & $1,934(90.1)$ & & $129(90.8)$ & $740(94.1)$ & \\
\hline ADGs, mean \pm SD & $10.4 \pm 3.49$ & $10.4 \pm 3.67$ & 0.775 & $11.3 \pm 3.52$ & $10.8 \pm 3.77$ & 0.117 \\
\hline Underlying conditions§ & & & & & & \\
\hline Asthma & $265(38.5)$ & $751(35.0)$ & 0.094 & $64(45.1)$ & $311(39.6)$ & 0.219 \\
\hline Bronchiectasis & $169(24.6)$ & $335(15.6)$ & $<0.001$ & $19(13.4)$ & $90(11.5)$ & 0.511 \\
\hline Chronic kidney disease & $40(5.8)$ & $199(9.3)$ & 0.004 & $12(8.5)$ & $66(8.4)$ & 0.983 \\
\hline COPD & $462(67.2)$ & $1,209(56.3)$ & $<0.001$ & $118(83.1)$ & $474(60.3)$ & $<0.001$ \\
\hline Cystic fibrosis & $\leq 5(\leq 0.7)$ & $\leq 5(\leq 0.2)$ & 0.327 & $\leq 5(\leq 3.5)$ & $\leq 5(\leq 0.6)$ & 0.172 \\
\hline Diabetes mellitus & $\overline{121}(17.6)$ & $518 \overline{(24.1)}$ & $<0.001$ & $\overline{28}(\overline{19} .7)$ & $206(26.2)$ & 0.101 \\
\hline GERD & $139(20.2)$ & 429 (20.0) & 0.903 & $31(21.8)$ & $158(20.1)$ & 0.638 \\
\hline HIV infection & $\leq 5(\leq 0.7)$ & $\leq 5(\leq 0.2)$ & 0.087 & 0 & 0 & NA \\
\hline Interstitial lung disease & $\overline{8} 1(11.8)$ & $138(6.4)$ & $<0.001$ & $14(9.9)$ & $62(7.9)$ & 0.430 \\
\hline Lung cancer & $19(2.8)$ & $135(6.3)$ & $<0.001$ & $9(6.3)$ & $74(9.4)$ & 0.237 \\
\hline Prior TB & $\leq 5(\leq 0.7)$ & $17(0.8)$ & 0.161 & $\leq 5(\leq 3.5)$ & $12(1.5)$ & 0.915 \\
\hline Rheumatoid arthritis & $27(3.9)$ & $79(3.7)$ & 0.770 & $6(4.2)$ & $31(3.9)$ & 0.875 \\
\hline
\end{tabular}

*Values are no. (\%) except as indicated. According to privacy regulations, values representing <6 persons are reported as $\leq 5$, and data are presented as a range of values for categorical variables where back-calculation is possible. ADGs, aggregated diagnostic groups (from the adjusted clinical group case mix system); COPD, chronic obstructive pulmonary disease; GERD, gastroesophageal reflux disease; MAC, Mycobacterium avium complex; M. xenopi; Mycobacterium xenopi; NA, not applicable; PD, pulmonary disease; TB, tuberculosis.

†Treated defined as $>60$ continuous days of treatment with $>1$ drugs/classes commonly used to treat MAC or $M$. xenopi PD (macrolide, ethambutol, rifamycin, fluoroquinolone, linezolid, inhaled amikacin, or, for $M$. xenopi, isoniazid), started within 1 y of any positive culture for the causative nontuberculous mycobacteria species/complex.

fDerived from rural index for Ontario group, a measure of rurality designed for Ontario (9).

§Defined according to inpatient and outpatient diagnostic codes in databases of hospital discharges and physicians' services claims, respectively; see Appendix (https://wwwnc.cdc.gov/EID/article/25/7/18-1817-App1.pdf) for definitions. Definitions have been validated for all underlying conditions with the exception of bronchiectasis and interstitial lung disease. 
We examined the proportion of patients for whom particular regimens had ever been prescribed for $\geq 60$ days during their first treatment episode. Among patients with treated MAC PD, the guidelines-recommended standard triple regimen (macrolide/ethambutol/rifamycin) was the most commonly prescribed (47.4\%), followed by nonmacrolide monotherapy $(29.2 \%)$ and macrolide monotherapy $(20.5 \%)$ (Table 2). Drug regimens associated with development of macrolide-resistant MAC (macrolide monotherapy, macrolide/fluoroquinolone, and macrolide/rifamycin) $(17,18)$ were prescribed for 224/688 (32.6\%) of MAC PD patients. Among $M$. xenopi PD patients who received treatment, standard triple therapy was prescribed for $35.9 \%$, followed by nonmacrolide monotherapy for $28.2 \%$, and macrolide monotherapy for $23.9 \%$ (Table 2).

The flow of antimicrobial drug treatment during the first 18 months (i.e., regimen sequence, duration, and transitions) revealed that, among MAC PD patients who

Table 2. Proportion of patients with MAC PD and M. xenopi PD who had ever received each antimicrobial drug and select drug combinations and duration during first treatment episode, Ontario, Canada, 2001-2013*

\begin{tabular}{|c|c|c|c|c|}
\hline \multirow[b]{2}{*}{ Treatment† } & \multicolumn{2}{|c|}{ MAC PD, $\mathrm{n}=688$} & \multicolumn{2}{|c|}{ M. xenopi PD, $\mathrm{n}=142$} \\
\hline & No. (\%) & Mean duration $\pm S D, d$ & No. (\%) & Mean duration $\pm \mathrm{SD}, \mathrm{d}$ \\
\hline \multicolumn{5}{|l|}{ Individual drug } \\
\hline \multicolumn{5}{|l|}{ Macrolide } \\
\hline Any & 599 (87.1) & $447 \pm 367$ & $120(84.5)$ & $359 \pm 312$ \\
\hline Clarithromycin & $318(46.2)$ & $369 \pm 364$ & $66(46.5)$ & $319 \pm 367$ \\
\hline Azithromycin & $354(51.5)$ & $424 \pm 348$ & $66(46.5)$ & $335 \pm 244$ \\
\hline \multicolumn{5}{|l|}{ Rifamycin } \\
\hline Any & $403(58.6)$ & $437 \pm 366$ & $69(48.6)$ & $349 \pm 228$ \\
\hline Rifampin & $384(55.8)$ & $434 \pm 363$ & $61(43.0)$ & $351 \pm 233$ \\
\hline Rifabutin & $30(4.4)$ & $323 \pm 285$ & $9(6.3)$ & $294 \pm 217$ \\
\hline Ethambutol & $483(70.2)$ & $456 \pm 357$ & $84(59.2)$ & $363 \pm 284$ \\
\hline \multicolumn{5}{|l|}{ Fluoroquinolone } \\
\hline Any & $232(33.7)$ & $369 \pm 353$ & $63(44.4)$ & $312 \pm 189$ \\
\hline Moxifloxacin & 82 (11.9) & $318 \pm 382$ & $27(19.0)$ & $251 \pm 195$ \\
\hline Levofloxacin & $56(8.1)$ & $226 \pm 239$ & $11(7.7)$ & $240 \pm 282$ \\
\hline Ciprofloxacin & $137(19.9)$ & $328 \pm 333$ & $36(25.4)$ & $283 \pm 181$ \\
\hline Gatifloxacin & $\leq 5(\leq 0.7)$ & $126 \pm 163$ & 0 & NA \\
\hline Norfloxacin & 8 (1.2) & $197 \pm 288$ & 0 & NA \\
\hline Linezolid & 0 & NA & 0 & NA \\
\hline Isoniazid & NA & NA & $\leq 5(\leq 3.5)$ & $160 \pm 110$ \\
\hline \multicolumn{5}{|l|}{ Drug regimen } \\
\hline $\begin{array}{l}\text { Standard triple: macrolide + ethambutol + } \\
\text { rifamycin } \pm \text { others }\end{array}$ & $326(47.4)$ & $369 \pm 269$ & $51(35.9)$ & $241 \pm 173$ \\
\hline Macrolide + ethambutol & $91(13.2)$ & $315 \pm 283$ & $11(7.7)$ & $159 \pm 83$ \\
\hline Macrolide + rifamycin & $49(7.1)$ & $284 \pm 392$ & $10(7.0)$ & $251 \pm 208$ \\
\hline Macrolide + fluoroquinolone & $65(9.4)$ & $267 \pm 278$ & $20(14.1)$ & $228 \pm 153$ \\
\hline Other macrolide-containing combinations $\ddagger$ & $115(16.7)$ & $346 \pm 276$ & $31(21.8)$ & $295 \pm 156$ \\
\hline Nonmacrolide combination§ & $63(9.2)$ & $258 \pm 298$ & $19(13.4)$ & $198 \pm 187$ \\
\hline Macrolide monotherapy & $141(20.5)$ & $262 \pm 358$ & $34(23.9)$ & $330 \pm 509$ \\
\hline Nonmacrolide monotherapy & $201(29.2)$ & $206 \pm 226$ & $40(28.2)$ & $253 \pm 207$ \\
\hline No. drugs given, mean \pm SD & $2.5 \pm 0.9$ & & $2.4 \pm 1.0$ & \\
\hline \multicolumn{5}{|l|}{ No. drugs given } \\
\hline 1 & $142(20.6)$ & & $37(26.1)$ & \\
\hline 2 & $121(17.6)$ & & 24 (16.9) & \\
\hline 3 & $372(54.1)$ & & $69(48.6)$ & \\
\hline 4 & $53(7.7)$ & & $12(8.5)$ & \\
\hline \multicolumn{5}{|l|}{ No. switched regimens } \\
\hline 0 & $401(58.3)$ & & $85(59.9)$ & \\
\hline 1 & $177(25.7)$ & & $33(23.2)$ & \\
\hline 2 & $72(10.5)$ & & $14(9.9)$ & \\
\hline$\geq 3$ & $38(5.5)$ & & $10(7.0)$ & \\
\hline $\begin{array}{l}\text { Maximum no. drugs used at any } 1 \text { time, mean } \\
\pm \mathrm{SD}\end{array}$ & $2.4 \pm 0.9$ & & $2.3 \pm 0.9$ & \\
\hline \multicolumn{5}{|c|}{$\begin{array}{l}\text { *MAC, Mycobacterium avium complex; M. xenopi, Mycobacterium xenopi; PD, pulmonary disease. } \\
\dagger \geq 60 \text { continuous d of treatment with } \geq 1 \text { drugs/classes commonly used to treat MAC PD or } M \text {. xenopi PD (mac } \\
\text { fluoroquinolone, linezolid, inhaled amikacin, or for } M \text {. xenopi PD, isoniazid), started within } 1 \text { y of any positive } c \\
\text { mycobacteria species/complex. Patients may have received }>1 \text { drug from an antibiotic class, and/or }>1 \text { antibioti } \\
\text { episode, so values do not add up to } 100 \% \text {. According to privacy regulations, values representing }<6 \text { persons a } \\
\text { as a range of values for categorical variables where back-calculation is possible. } \\
\ddagger \geq 2 \text { drugs excluding: macrolide }+ \text { fluoroquinolone, macrolide }+ \text { EMB, macrolide + rifamycin. } \\
\S \geq 2 \text { drugs (e.g., ethambutol, a rifamycin, a fluoroquinolone (or for } M \text {. xenopi PD, isoniazid), without macrolide. } \\
\uparrow \text { Change in treatment lasting } \geq 60 \mathrm{~d} \text {, in the first treatment episode. }\end{array}$} \\
\hline
\end{tabular}


received treatment, the most common starting regimen was standard triple therapy $(290 / 688 ; 42.1 \%)$ (Figure), prescribed for a mean $( \pm \mathrm{SD})$ of $315( \pm 167)$ days (median 334; IQR 151-467 days) before a regimen switch or discontinuation. Among MAC PD patients for whom the initial regimen was associated with development of macrolide resistance, these regimens were prescribed for the following mean $( \pm \mathrm{SD})$ durations before a switch or discontinuation: macrolide monotherapy, 230 ( \pm 167$)$ days; macrolide/fluoroquinolone, $216( \pm 147)$ days; and macrolide/ rifamycin, $197( \pm 139)$ days (Figure). For a large minority of MAC PD patients, therapy was switched during the first treatment episode; $\geq 1$ regimen was switched for $41.7 \%$ (Table 2; Figure). Among MAC PD patients who received treatment, $50.2 \%$ received treatment for $\geq 12$ months before discontinuation. Among M. xenopi PD patients who received treatment, $31.0 \%$ initially received standard triple therapy and $40.1 \%$ underwent $\geq 1$ regimen switch (Table 2 ).

Among MAC PD patients who received treatment, for their first regimen, the specialties of the main prescribing physicians varied. The prescriber was a pulmonologist for $55.7 \%$, an infectious diseases specialist for $10.0 \%$, an internal medicine specialist for $7.4 \%$, a family physician/ general practitioner for $12.3 \%$, and another specialist or of unknown specialty for $14.5 \%$ (Table 3 ).

According to bivariate analyses, patients with MAC PD whose initial regimen was macrolide monotherapy were more likely than those whose initial regimen was anything else to have asthma or COPD , to have received a long-acting bronchodilator or oral corticosteroid in the prior year, to have visited an emergency department or been hospitalized in the prior 2 years for an asthma or COPD exacerbation, to have received oxygen at home, and to have received pulmonary function tests in the previous 5 years (Table 4). However, according to adjusted analyses, only use of oral corticosteroids in the prior year was significantly associated with a starting regimen of macrolide monotherapy (adjusted odds ratio 2.01, 95\% CI 1.16-3.50).

\section{Discussion}

In this population-based study of treatment practices for MAC PD and M. xenopi PD in adults $\geq 66$ years of age, we found that a minority of patients received antimicrobial therapy: $24 \%$ of MAC PD patients and $15 \%$ of

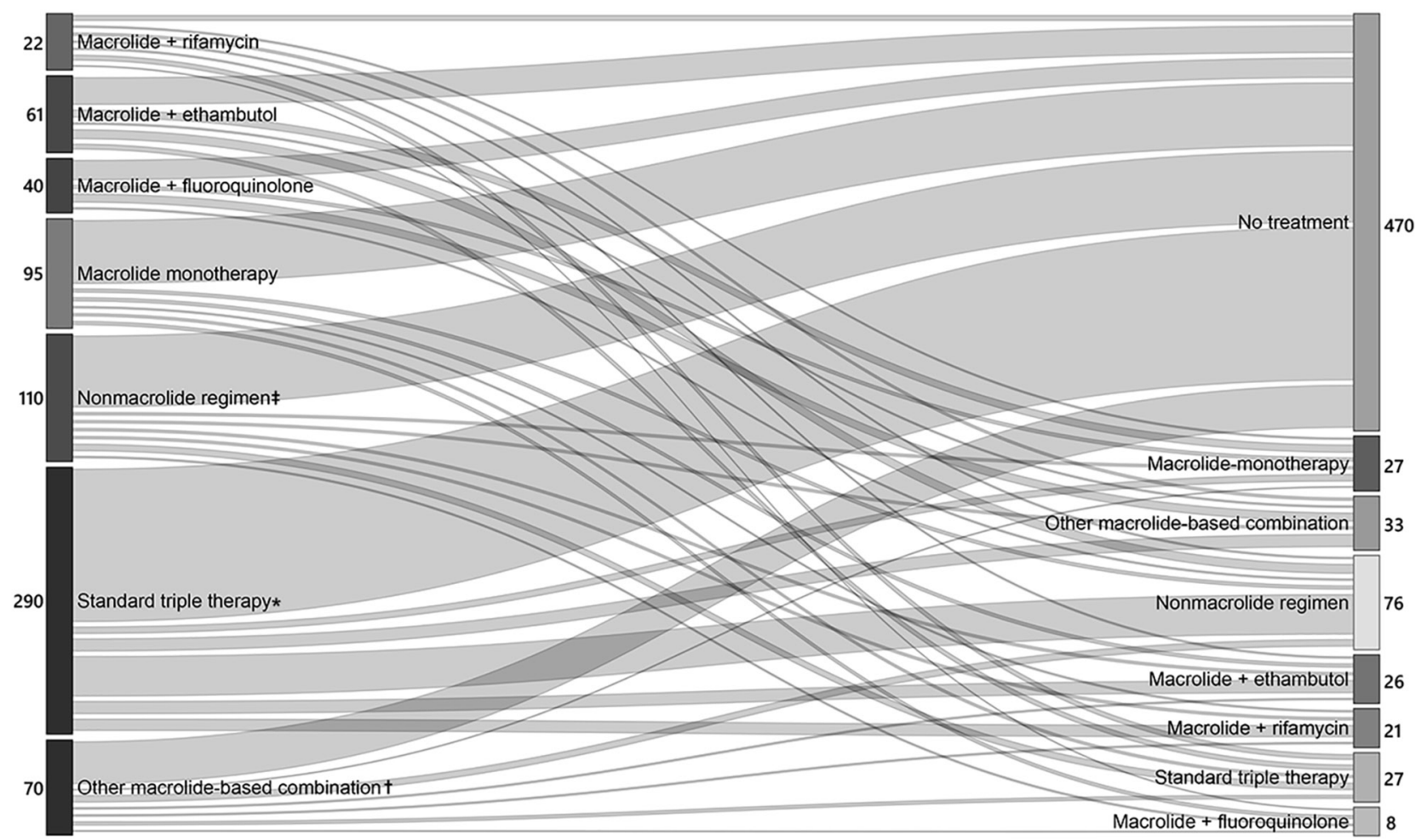

Figure. Flow of therapy for 688 patients with Mycobacterium avium complex pulmonary disease, depicting transition between first and second regimens during first 18 months of treatment, Ontario, Canada, 2001-2013. Values are the number of patients receiving each treatment regimen in each epoch of therapy. An epoch is defined as $\geq 60$ days of the therapy. The width of the lines is proportional to the number of patients receiving and transitioning between each regimen. Mean $( \pm S D)$ duration of treatment, in days, for each starting regimen is as follows: standard triple therapy 315 ( \pm 167$)$, other macrolide containing combination 331 ( \pm 157 ), macrolide-ethambutol 274 ( \pm 172$)$, macrolide monotherapy 230 ( \pm 167$)$, nonmacrolide containing regimen $176( \pm 178)$, macrolide-fluoroquinolone 216 ( \pm 147$)$, macrolide-rifamycin 197 ( \pm 139). *Macrolide, ethambutol, and a rifamycin, \pm other drugs; †macrolide $+\geq 2$ additional drugs (other than standard triple therapy); łethambutol, a rifamycin, or fluoroquinolone, either alone or in combination. 
Table 3. Initial treatment regimen, by prescriber specialty, for 688 patients with Mycobacterium avium complex pulmonary disease, Ontario, Canada, 2001-2013*

\begin{tabular}{|c|c|c|c|c|c|}
\hline \multirow[b]{2}{*}{ Regimen } & \multicolumn{5}{|c|}{ Specialty, no. (\%) patients } \\
\hline & $\begin{array}{c}\text { Respirology, } \\
\mathrm{n}=383(55.7)\end{array}$ & ID, $n=69(10.0)$ & $\mathrm{GIM}, \mathrm{n}=51(7.4)$ & $\begin{array}{c}\text { FP/GP, } n=85 \\
(12.3)\end{array}$ & $\begin{array}{c}\text { Other/unknown, } \\
n=100(14.5)\end{array}$ \\
\hline Standard triple therapy & $166(43.3)$ & $37(53.6)$ & $22(43.1)$ & $34(40.0)$ & $31(31.0)$ \\
\hline Macrolide monotherapy & $55(14.4)$ & $7(10.1)$ & $\leq 5(\leq 9.8)$ & $14(16.5)$ & $15(15.0)$ \\
\hline $\begin{array}{l}\text { Macrolide + rifamycin or } \\
\text { fluoroquinolone }\end{array}$ & $38(9.9)$ & $\leq 5(\leq 7.2)$ & $\leq 5(\leq 9.8)$ & $6(7.1)$ & $8(8.0)$ \\
\hline Other & $124(32.4)$ & 20-25 (29.0-36.1) & $20(39.2)$ & $31(36.5)$ & $46(46.0)$ \\
\hline
\end{tabular}

M. xenopi PD patients. During the first treatment episode, the most commonly prescribed regimen, initially and overall, was standard triple therapy. However, it is concerning that many MAC PD patients received $\geq 60$ days of treatment with regimens associated with macrolide resistance, a situation that is extremely difficult to treat and associated with high mortality rates (17-19). Macrolide monotherapy was prescribed for $20 \%$ of MAC PD patients, and other regimens associated with facilitating macrolide resistance (macrolide/fluoroquinolone, macrolide/rifamycin) were also frequently prescribed. Although standard triple therapy was prescribed initially for $42 \%$ and ever (during the first treatment episode) for $47 \%$ of patients, regimens that facilitate macrolide resistance were prescribed initially for $23 \%$ and ever for $33 \%$. Treatment flow was complex, and switches between regimens were common.

In our study, the proportion of patients with MAC PD who received antimicrobial drug treatment $(24 \%)$ was lower than that described by others. Studies from South Korea (20) and Germany (21) reported treatment rates within 3 years of diagnosis of $65 \%$ for MAC PD and $74 \%$ for NTM PD patients. In Oregon, USA, treatment was initiated within 2 years of diagnosis for $54 \%$ of NTM PD patients (22). According to physician survey studies, antimicrobial drug treatment was received by $55 \%$ of MAC PD patients in the United States (5), 68\% of NTM PD patients from 5 countries in the European Union (6), and $43 \%$ of NTM PD patients in Japan (6). The reasons why a relatively small proportion of MAC PD patients in our study received treatment are probably many. First, our definition of NTM PD (based on microbiological criteria only) probably more often misclassified patients from whom NTM were repeatedly isolated as having disease, compared with the Oregon study, which reviewed all diagnostic criteria (22), and the Germany study, which used diagnostic codes (21). Second, our study was population based and thereby included the full spectrum of disease severity and physician expertise, compared with specialty clinic-based studies (20), which probably comprise patients with more severe disease and physicians who may be more likely to treat NTM PD because of greater experience. Third, we included only adults $\geq 66$ years of age; older patients may be less likely to receive treatment, as was noted among the MAC PD patients in this study and has been described by others (20). Some patients may not have been prescribed treatment because of a limited life expectancy resulting from underlying conditions; however, when we performed a sensitivity analysis limited to patients who survived $\geq 2$ years after NTM PD diagnosis, we found no notable change in the proportion who received treatment. Fourth, we required 1 positive culture for the causative NTM within 1 year of treatment initiation and $\geq 60$ continuous days of dispensed prescriptions, which were not requirements for the other studies. Of note, the proportion of patients who received treatment in our study was similar to the $18 \%$ of NTM PD patients who received treatment at 4 integrated US healthcare delivery systems (23), in which patients were identified by using a combination of culture results and codes from the International Classification of Diseases, Ninth Revision.

We found associations between baseline characteristics and receipt of MAC PD treatment. Income distribution was significantly associated with treatment; patients residing in neighborhoods in lower income quintiles seemed less likely to receive treatment. Although Canada provides universal access to medically necessary health services, including prescription drugs for adults $\geq 65$ years of age, socioeconomic disparities in access to specialist care have been observed (24) and may play a role. We also found that patients living in urban settings were less likely to receive treatment; this finding is somewhat surprising in that others have shown that urban patients are more likely than rural patients to receive ambulatory care, including specialist care, for other chronic medical conditions $(25,26)$. Whether a disparity in the proportion of patients with true disease exists when comparing patients with MAC isolates from urban versus rural settings is not clear.

We found prescription of standard triple therapy for MAC PD to be more common ( $47 \%$ ever received it and $42 \%$ received it as initial therapy) than that reported in the United States (13\% ever received) (5), the European Union ( $9 \%$ for $>6$ months) (6), and Germany (19\%) (21) but similar to that reported in Japan ( $42 \%$ for $>6$ months) (b). The possible reasons for these differences include differences in study methods, prescribing physician specialty, 
Treatment of Mycobacterium Pulmonary Disease

Table 4. Characteristics of MAC PD patients according to initial treatment regimen, Ontario, Canada, 2001-2013*

\begin{tabular}{|c|c|c|c|c|c|c|}
\hline Characteristic & $\begin{array}{c}\text { Macrolide } \\
\text { monotherapy, } \mathrm{n}=95 \dagger\end{array}$ & $\begin{array}{c}\text { Other regimen, } \\
n=593\end{array}$ & $\begin{array}{l}\text { Unadjusted OR } \\
(95 \% \mathrm{Cl})\end{array}$ & $p$ value & $\begin{array}{c}\text { Adjusted OR } \\
(95 \% \mathrm{Cl}) \ddagger\end{array}$ & $\mathrm{p}$ value \\
\hline \multicolumn{7}{|l|}{ Sex } \\
\hline $\mathrm{F}$ & $52(54.7)$ & $357(60.2)$ & $0.80(0.52-1.24)$ & 0.314 & $0.92(0.57-1.48)$ & 0.738 \\
\hline M & $43(45.3)$ & $236(39.8)$ & Referent & NA & Referent & NA \\
\hline Age, mean \pm SD & $76.21 \pm 6.67$ & $75.52 \pm 5.81$ & $1.02(0.98-1.06)$ & 0.292 & $1.03(0.99-1.07)$ & 0.123 \\
\hline \multicolumn{7}{|l|}{ Income quintile } \\
\hline 1 (lowest) & $19(20.0)$ & $123(20.7)$ & Referent & 0.682 & NA & NA \\
\hline 2 & $22(23.2)$ & $124(20.9)$ & $1.15(0.59-2.23)$ & 0.470 & NA & NA \\
\hline 3 & $13(13.7)$ & $111(18.7)$ & $0.76(0.36-1.61)$ & 0.945 & NA & NA \\
\hline 4 & $16(16.8)$ & $101(17.0)$ & $1.03(0.50-2.10)$ & 0.752 & NA & NA \\
\hline 5 (highest) & $21-25(22.1-26.3)$ & $134(22.6)$ & $1.11(0.58-2.14)$ & 0.986 & NA & NA \\
\hline Missing data & $\leq 5(\leq 2.1)$ & 0 & NA & & NA & NA \\
\hline \multicolumn{7}{|l|}{ Residency§ } \\
\hline Rural & $\leq 5(\leq 2.1)$ & $35(5.9)$ & $0.33(0.08-1.40)$ & 0.132 & NA & NA \\
\hline Suburban & $8-1 \overline{2}(8.4-12.6)$ & $79(13.3)$ & $0.73(0.36-1.47)$ & 0.378 & NA & NA \\
\hline Urban & $83(87.4)$ & $479(80.8)$ & Referent & NA & NA & NA \\
\hline ADGs, mean \pm SD & $10.45 \pm 3.90$ & $10.36 \pm 3.42$ & $1.01(0.95-1.07)$ & 0.805 & NA & NA \\
\hline \multicolumn{7}{|l|}{ Underlying conditions } \\
\hline Asthma & $47(49.5)$ & $218(36.8)$ & $1.68(1.09-2.60)$ & 0.019 & $1.22(0.72-2.07)$ & 0.451 \\
\hline Bronchiectasis & $27(28.4)$ & $142(23.9)$ & $1.26(0.78-2.05)$ & 0.347 & $1.19(0.71-1.99)$ & 0.504 \\
\hline Chronic kidney disease & $7(7.4)$ & $133(5.6)$ & $1.35(0.58-3.15)$ & 0.486 & NA & NA \\
\hline COPD & 75 (78.9) & $387(65.3)$ & $2.00(1.19-3.36)$ & 0.009 & $1.47(0.81-2.66)$ & 0.208 \\
\hline Diabetes mellitus & $21(22.1)$ & $100(16.9)$ & $1.40(0.82-2.38)$ & 0.214 & NA & NA \\
\hline GERD & $21(22.1)$ & $118(19.9)$ & $1.14(0.68-1.93)$ & 0.619 & NA & NA \\
\hline Interstitial lung disease & $14(14.7)$ & $67(11.3)$ & $1.36(0.73-2.53)$ & 0.335 & NA & NA \\
\hline Lung cancer & $\leq 5(\leq 2.1)$ & $17(2.9)$ & $0.73(0.17-3.21)$ & 0.676 & NA & NA \\
\hline Rheumatoid arthritis & $\leq 5(<2.1)$ & $25(4.2)$ & $0.49(0.11-2.10)$ & 0.335 & NA & NA \\
\hline \multicolumn{7}{|l|}{ Drug exposure within $1 \mathrm{y \#}$} \\
\hline Short-acting BD & $49(51.6)$ & $271(45.7)$ & $1.27(0.82-1.95)$ & 0.287 & $0.69(0.38-1.26)$ & 0.225 \\
\hline Long-acting BD & $52(54.7)$ & $248(41.8)$ & $1.68(1.09-2.60)$ & 0.019 & $1.16(0.56-2.39)$ & 0.694 \\
\hline ICS & $55(57.9)$ & $287(48.4)$ & $1.47(0.95-2.27)$ & 0.087 & $0.89(0.42-1.87)$ & 0.754 \\
\hline OCS & $38(40.0)$ & $130(21.9)$ & $2.37(1.51-3.74)$ & $<0.001$ & $2.01(1.16-3.50)$ & 0.013 \\
\hline Methylxanthine & $9(9.5)$ & $28(4.7)$ & $2.11(0.96-4.63)$ & 0.062 & $1.52(0.64-3.57)$ & 0.340 \\
\hline $\begin{array}{l}\text { ED visit/hospitalization for } \\
\text { asthma or COPD within } 2 \text { y\# }\end{array}$ & $22(23.2)$ & $88(14.8)$ & $1.73(1.02-2.93)$ & 0.042 & $0.92(0.48-1.77)$ & 0.799 \\
\hline $\begin{array}{l}\text { Prior/current home oxygen } \\
\text { therapy }\end{array}$ & $12(12.6)$ & $31(5.2)$ & $2.62(1.30-5.31)$ & 0.007 & $1.83(0.84-3.98)$ & 0.128 \\
\hline PFTs within 5 y\# & $78(82.1)$ & $416(70.2)$ & $1.95(1.12-3.39)$ & 0.018 & $1.52(0.82-2.78)$ & 0.180 \\
\hline Pulmonologist prescriber & $55(57.9)$ & $328(55.3)$ & $1.11(0.72-1.72)$ & 0.638 & $1.03(0.66-1.63)$ & 0.889 \\
\hline \multicolumn{7}{|c|}{$\begin{array}{l}\text { *Values are no. (\%) except as indicated. According to privacy regulations, values representing }<6 \text { persons are reported as } \leq 5 \text {, and data are presented as } \\
\text { a range of values for categorical variables where back-calculation is possible. ADGs, aggregated diagnostic groups (from the adjusted clinical group case } \\
\text { mix system); BD, bronchodilator; COPD, chronic obstructive pulmonary disease; ED, emergency department; GERD, gastroesophageal reflux disease; } \\
\text { ICS, inhaled corticosteroid; MAC, Mycobacterium avium complex; NA, not applicable; NTM, nontuberculous mycobacteria; OCS, oral corticosteroid; OR, } \\
\text { odds ratio; PD, pulmonary disease; PFTs, pulmonary function tests. } \\
\text { †Macrolide monotherapy was the first antibiotic regimen given after NTM PD diagnosis, and was considered } \geq 60 \mathrm{~d} \text { with no companion drugs of interest. } \\
\text { †Variables were selected for inclusion in the multivariable model a priori, based on clinical relevance. } \\
\text { §Derived from rural index for Ontario group, a measure of rurality designed for Ontario (9). } \\
\text { TDefined according to inpatient and outpatient diagnostic codes in databases of hospital discharges and physicians' services claims, respectively. } \\
\text { Definitions have been validated for all underlying conditions with the exception of bronchiectasis and interstitial lung disease. } \\
\text { \#Before NTM PD diagnosis. }\end{array}$} \\
\hline
\end{tabular}

financial coverage for medications, and familiarity with ATS/IDSA guidelines. Regarding physician specialties, the proportion of MAC PD patients receiving treatment from a pulmonologist in our study (57\%) was similar to that in Japan (54\%) (6) but higher than that in the European Union (29\%) (6) and in the United States (37\%) (5). Pulmonologists may be more aware of the ATS/IDSA guidelines than are other specialists. However, pulmonologists in Ontario $(43 \%)$ seemed more likely than those in the United States $(18 \%)$ to prescribe standard triple therapy for MAC PD (5), which may result from different patient populations and medication coverage. We included only adults $\geq 66$ years of age because this population has comprehensive medication coverage. Pulmonologists in the United States may prescribe nonstandard antimicrobial drug regimens for patients who do not have prescription drug coverage because of cost.

In our study, $20 \%$ of MAC PD patients who received treatment were prescribed $\geq 60$ days of macrolide monotherapy, $9 \% \geq 60$ days of macrolide/fluoroquinolone, and $7 \% \geq 60$ days of macrolide/rifamycin therapy. Findings of studies of physicians in the United States (5) and Germany (21) were similar. These regimens are associated with development of macrolide resistance $(17,18)$; resistance developed in $20 \%$ of 59 patients who received macrolide monotherapy for 4 months compared with $4 \%$ 
of 303 patients who received standard triple therapy (17). In our study, among 95 MAC PD patients whose initial regimen was macrolide monotherapy, the mean duration was 230 days; macrolide/fluoroquinolone and macrolide/ rifamycin regimens were given for similar durations. Therefore, the regimen duration was long enough to constitute a risk for macrolide resistance.

For some patients, these drugs may have been prescribed for other conditions. This possibility applies especially to macrolide monotherapy, which may have been prescribed to treat exacerbations of asthma, COPD, or bronchiectasis and may not have been prescribed to treat MAC per se. This possibility is supported by our analyses; bivariate analysis indicated that presence of asthma and COPD were associated with receipt of macrolide monotherapy versus another regimen. Although bronchiectasis was not associated with receipt of macrolide monotherapy, our databases contain no validated bronchiectasis definition, and the definition we used (1 physician billing claim or hospitalization with bronchiectasis diagnosis) seems to be of very low sensitivity, given the small number of patients with NTM assigned a code for bronchiectasis. Also, some treatments for asthma/COPD (long-acting bronchodilators, oral corticosteroids, home oxygen), as well as emergency department visits/hospitalizations for asthma/ COPD, were associated with prescription of macrolide monotherapy. According to multivariable analyses, the only variable associated with prescription of macrolide monotherapy was receipt of oral corticosteroids $\leq 1$ year before MAC PD diagnosis, which is consistent with the possibility that macrolide monotherapy was prescribed to prevent asthma/COPD exacerbations. It is possible that some patients for whom macrolide monotherapy was prescribed for asthma, COPD, or bronchiectasis did not have clinical or radiologic findings of MAC PD. However, these patients did fulfill microbiological criteria for MAC PD and had $\geq 1$ positive culture within 1 year before filling the prescription. No data describe the risk of inducing macrolide resistance in persons with positive sputum cultures who do not meet full diagnostic criteria for MAC PD. However, the fact that one fifth of patients in our study who received treatment and met microbiological criteria for MAC PD received this regimen for $\geq 60$ days is concerning. Given the increasing use of macrolides for asthma, COPD, and bronchiectasis, further research into the safety of these drugs in patients with NTM isolation is needed.

Few data exist regarding prescribing patterns for patients with M. xenopi PD. A retrospective study of 136 patients in France who had M. xenopi PD meeting full ATS/ IDSA diagnostic criteria found that $59 \%$ received treatment (27) compared with $15 \%$ in our study. That study found that patients' initial treatment regimens contained an average of 4 drugs among rifamycins (88\%), ethambutol $(75 \%)$, isoniazid
(66\%), clarithromycin (30\%), and fluoroquinolones (21\%). In our study, patients received fewer drugs (mean $2.4 \pm 1.0$ SD) and were more likely to receive a macrolide $(84 \%)$ or a fluoroquinolone (44\%) and less likely to receive a rifamycin (49\%), ethambutol $(59 \%)$, or isoniazid $(\leq 4 \%)$. The difference between prescribing patterns for $M$. xenopi $\mathrm{PD}$ in France versus Ontario may be partially explained by the periods of the studies (1983-2003 in France vs. 2001-2013 in Ontario); evidence supporting the efficacy of macrolides for treating $M$. xenopi infection emerged in the mid-1990s $(28-31)$. Also, the study in France was not restricted to older adults and included only patients at 1 of 13 hospitals, which may have limited them to more severe cases. Another possible explanation relates to differences in the more geographically proximal treatment guidelines; the 1999 British Thoracic Society guidelines recommended treatment with rifampin and ethambutol \pm isoniazid (32), whereas the 1997 and 2007 ATS/IDSA guidelines recommend a regimen of clarithromycin, rifampin, and ethambutol $(4,28), \pm$ isoniazid \pm moxifloxacin (4).

For MAC PD and M. xenopi PD patients who received treatment, regimen switches were common $(42 \%$ of MAC PD patients underwent $\geq 1$ regimen switch). Others have reported similar findings; in 2 case series of MAC PD patients who received standard triple therapy, regimens were switched for $46 \%-71 \%$ of patients receiving daily therapy and $3 \%-21 \%$ of patients receiving intermittent therapy $(33,34)$. We did not study daily versus intermittent therapy and are not able to draw conclusions regarding tolerability of different drugs or combinations. However, our finding of frequent regimen switches suggests that drug intolerance was common and may partially explain the frequent use of regimens not recommended in treatment guidelines.

Our study has several limitations. We based our definition of NTM PD on microbiological criteria alone and therefore probably misclassified some patients as having true disease, possibly contributing to the observation that a low proportion of patients received treatment. We defined treatment as $\geq 60$ days of an antimicrobial drug of interest being dispensed; this definition may not capture some patients in whom there was an intent to treat, such as patients who had medications prescribed but never dispensed and patients who received treatment for NTM PD but stopped taking the medication in $<60$ days. Because we included only adults $\geq 66$ years of age, our findings may not apply to younger patients. Last, we were not able to study the use of clofazimine or injectable aminoglycosides because the relevant information is not contained in our databases. This omission may have caused us to erroneously label some patients as having received an inappropriate regimen when the regimen was strengthened by clofazimine or an aminoglycoside. However, because 
clofazimine is not approved for use in Canada and was difficult to access during the study period, we think that these patients are probably few.

The use of inhaled amikacin seemed to be rare. We also excluded patients who met diagnostic criteria for NTM PD associated with $>1$ species. The proportion was small, combined with exclusions for TB amounting to $10.4 \%$ for MAC PD and $11.1 \%$ for $M$. xenopi PD. Given the species distribution of NTM in Ontario (MAC PD and M. xenopi PD comprising the overwhelming majority of treated NTM episodes), the very high similarity between MAC PD and $M$. xenopi PD treatments, and our lack of data for intravenous treatments needed to analyze $M$. abscessus therapy, we elected to exclude these patients.

In summary, the most commonly prescribed regimen for MAC PD and M. xenopi PD in Ontario was standard triple therapy. This finding is somewhat reassuring; however, a large minority of patients with MAC PD received regimens that may lead to macrolide resistance. Physicians who treat patients with NTM PD should take care to follow established treatment guidelines for management of this condition.

\section{Acknowledgment}

We thank IMS Brogan Inc. for use of their Drug Information Database.

This study was supported by ICES and Public Health Ontario, which are funded by annual grants from the Ontario Ministry of Health and Long-Term Care. The opinions, results, and conclusions reported in this article are those of the authors and are independent from the funding sources. No endorsement by ICES or the Ontario Ministry of Health and Long-Term Care is intended or should be inferred. Parts of this material are based on data and/or information compiled and provided by the Canadian Institute of Health Information. However, the analyses, conclusions, opinions, and statements expressed herein are those of the authors, and not necessarily those of the Institute.

\section{About the Author}

Dr. Brode is an assistant professor of medicine at the University of Toronto, the physician lead of the TB Program at West Park Healthcare Centre (Toronto), and a staff pulmonologist and a clinician in the NTM and TB Programs at Toronto Western Hospital (Toronto). Her research focuses on epidemiology, treatment, and outcomes for patients with TB and NTM infection.

\section{References}

1. Marras TK, Mendelson D, Marchand-Austin A, May K, Jamieson FB. Pulmonary nontuberculous mycobacterial disease, Ontario, Canada, 1998-2010. Emerg Infect Dis. 2013;19:1889-91. http://dx.doi.org/10.3201/eid1911.130737

2. Henkle E, Hedberg K, Schafer S, Novosad S, Winthrop KL. Population-based incidence of pulmonary nontuberculous mycobacterial disease in Oregon 2007 to 2012. Ann Am Thorac Soc. 2015;12:642-7. http://dx.doi.org/10.1513/ AnnalsATS.201412-559OC

3. Adjemian J, Olivier KN, Seitz AE, Holland SM, Prevots DR. Prevalence of nontuberculous mycobacterial lung disease in U.S. Medicare beneficiaries. Am J Respir Crit Care Med. 2012;185: 881-6. http://dx.doi.org/10.1164/rccm.201111-2016OC

4. Griffith DE, Aksamit T, Brown-Elliott BA, Catanzaro A, Daley C, Gordin F, et al.; ATS Mycobacterial Diseases Subcommittee; American Thoracic Society; Infectious Disease Society of America. An official ATS/IDSA statement: diagnosis, treatment, and prevention of nontuberculous mycobacterial diseases. Am J Respir Crit Care Med. 2007;175:367-416. http://dx.doi.org/10.1164/ rccm.200604-571ST

5. Adjemian J, Prevots DR, Gallagher J, Heap K, Gupta R, Griffith D. Lack of adherence to evidence-based treatment guidelines for nontuberculous mycobacterial lung disease. Ann Am Thorac Soc. 2014;11:9-16. http://dx.doi.org/10.1513/ AnnalsATS.201304-085OC

6. van Ingen J, Wagner D, Gallagher J, Morimoto K, Lange C, Haworth CS, et al.; NTM-NET. Poor adherence to management guidelines in nontuberculous mycobacterial pulmonary diseases. Eur Respir J. 2017;49:1601855. http://dx.doi. org/10.1183/ 13993003.01855-2016

7. Brode SK, Jamieson FB, Ng R, Campitelli MA, Kwong JC, Paterson JM, et al. Risk of mycobacterial infections associated with rheumatoid arthritis in Ontario, Canada. Chest. 2014;146:563-72. http://dx.doi.org/10.1378/chest.13-2058

8. Al Houqani M, Jamieson F, Chedore P, Mehta M, May K, Marras TK. Isolation prevalence of pulmonary nontuberculous mycobacteria in Ontario in 2007. Can Respir J. 2011;18:19-24. http://dx.doi.org/10.1155/2011/865831

9. Kralj B. Measuring "rurality" for purposes of health-care planning: an empirical measure for Ontario. Ontario Medical Review. 2000;10:33-52.

10. Hux JE, Ivis F, Flintoft V, Bica A. Diabetes in Ontario: determination of prevalence and incidence using a validated administrative data algorithm. Diabetes Care. 2002;25:512-6. http://dx.doi.org/10.2337/diacare.25.3.512

11. Lopushinsky SR, Covarrubia KA, Rabeneck L, Austin PC, Urbach DR. Accuracy of administrative health data for the diagnosis of upper gastrointestinal diseases. Surg Endosc. 2007; 21:1733-7. http://dx.doi.org/10.1007/s00464-006-9136-1

12. Widdifield J, Bombardier C, Bernatsky S, Paterson JM, Green D, Young J, et al. An administrative data validation study of the accuracy of algorithms for identifying rheumatoid arthritis: the influence of the reference standard on algorithm performance. BMC Musculoskelet Disord. 2014;15:216. http://dx.doi.org/10.1186/1471-2474-15-216

13. Reid RJ, MacWilliam L, Verhulst L, Roos N, Atkinson M. Performance of the ACG case-mix system in two Canadian provinces. Med Care. 2001;39:86-99. http://dx.doi.org/10.1097/ 00005650-200101000-00010

14. Gershon AS, Wang C, Guan J, Vasilevska-Ristovska J, Cicutto L, To T. Identifying patients with physician-diagnosed asthma in health administrative databases. Can Respir J. 2009;16:183-8. http://dx.doi.org/10.1155/2009/963098

15. Gershon AS, Wang C, Guan J, Vasilevska-Ristovska J, Cicutto L, To T. Identifying individuals with physician diagnosed COPD in health administrative databases. COPD. 2009;6:388-94. http://dx.doi.org/10.1080/15412550903140865

16. Fleet JL, Dixon SN, Shariff SZ, Quinn RR, Nash DM, Harel Z, et al. Detecting chronic kidney disease in population-based administrative databases using an algorithm of hospital encounter and physician claim codes. BMC Nephrol. 2013;14:81. http://dx.doi.org/10.1186/1471-2369-14-81 
17. Griffith DE, Brown-Elliott BA, Langsjoen B, Zhang Y, Pan X, Girard W, et al. Clinical and molecular analysis of macrolide resistance in Mycobacterium avium complex lung disease. Am J Respir Crit Care Med. 2006;174:928-34. http://dx.doi.org/10.1164/ rccm.200603-450OC

18. Morimoto K, Namkoong H, Hasegawa N, Nakagawa T, Morino E, Shiraishi Y, et al.; Nontuberculous Mycobacteriosis Japan Research Consortium. Macrolide-resistant Mycobacterium avium complex lung disease: analysis of 102 consecutive cases. Ann Am Thorac Soc. 2016;13:1904-11. http://dx.doi.org/10.1513/ AnnalsATS.201604-246OC

19. Moon SM, Park HY, Kim SY, Jhun BW, Lee H, Jeon K, et al. Clinical characteristics, treatment outcomes, and resistance mutations associated with macrolide-resistant Mycobacterium avium complex lung disease. Antimicrob Agents Chemother. 2016;60:6758-65. http://dx.doi.org/10.1128/AAC.01240-16

20. Hwang JA, Kim S, Jo KW, Shim TS. Natural history of Mycobacterium avium complex lung disease in untreated patients with stable course. Eur Respir J. 2017;49:1600537. http://dx.doi.org/10.1183/13993003.00537-2016

21. Diel R, Jacob J, Lampenius N, Loebinger M, Nienhaus A, Rabe KF, et al. Burden of non-tuberculous mycobacterial pulmonary disease in Germany. Eur Respir J. 2017;49:1602109. http://dx.doi.org/10.1183/13993003.02109-2016

22. Henkle E, Novosad SA, Shafer S, Hedberg K, Siegel SAR, Ku J, et al. Long-term outcomes in a population-based cohort with respiratory nontuberculous mycobacteria isolation. Ann Am Thorac Soc. 2017;14:1120-8. http://dx.doi.org/10.1513/ AnnalsATS.201610-8010C

23. Prevots DR, Shaw PA, Strickland D, Jackson LA, Raebel MA, Blosky MA, et al. Nontuberculous mycobacterial lung disease prevalence at four integrated health care delivery systems. Am J Respir Crit Care Med. 2010;182:970-6. http://dx.doi.org/10.1164/ rccm.201002-0310OC

24. Dunlop S, Coyte PC, McIsaac W. Socio-economic status and the utilisation of physicians' services: results from the Canadian National Population Health Survey. Soc Sci Med. 2000 51:123-33.

25. Tran C, Wijeysundera HC, Qui F, Tu JV, Bhatia RS. Comparing the ambulatory care and outcomes for rural and urban patients with chronic ischemic heart disease: a population-based cohort study. Circ Cardiovasc Qual Outcomes. 2014;7:835-43. http://dx.doi.org/ 10.1161/CIRCOUTCOMES.114.001076

26. Benchimol EI, Kuenzig ME, Bernstein CN, Nguyen GC, Guttmann A, Jones JL, et al.; Canadian Gastro-Intestinal
Epidemiology Consortium. Rural and urban disparities in the care of Canadian patients with inflammatory bowel disease: a population-based study. Clin Epidemiol. 2018;10:1613-26. http://dx.doi.org/10.2147/CLEP.S178056

27. Andréjak C, Lescure FX, Pukenyte E, Douadi Y, Yazdanpanah Y, Laurans G, et al.; Xenopi Group. Mycobacterium xenopi pulmonary infections: a multicentric retrospective study of 136 cases in north-east France. Thorax. 2009;64:291-6. http://dx.doi.org/10.1136/thx.2008.096842

28. Medical Section of the American Lung Association. Diagnosis and treatment of disease caused by nontuberculous mycobacteria. Am J Respir Crit Care Med. 1997;156:S1-25.

29. Klemens SP, Cynamon MH. Activities of azithromycin and clarithromycin against nontuberculous mycobacteria in beige mice. Antimicrob Agents Chemother. 1994;38:1455-9. http://dx.doi.org/10.1128/AAC.38.7.1455

30. Roche B, Rozenberg S, Cambau E, Desplaces N, Dion E, Dubourg G, et al. Efficacy of combined clarithromycin and sparfloxacin therapy in a patient with discitis: due to Mycobacterium xenopi. Rev Rhum Engl Ed. 1997;64:64-5.

31. Schmitt H, Schnitzler N, Riehl J, Adam G, Sieberth HG, Haase G. Successful treatment of pulmonary Mycobacterium xenopi infection in a natural killer cell-deficient patient with clarithromycin, rifabutin, and sparfloxacin. Clin Infect Dis. 1999;29:120-4. http://dx.doi.org/10.1086/520140

32. Subcommittee of the Joint Tuberculosis Committee of the British Thoracic Society. Management of opportunist mycobacterial infections: Joint Tuberculosis Committee Guidelines 1999. Thorax. 2000;55:210-8. http://dx.doi.org/ 10.1136/thorax.55.3.210

33. Wallace RJ Jr, Brown-Elliott BA, McNulty S, Philley JV, Killingley J, Wilson RW, et al. Macrolide/azalide therapy for nodular/bronchiectatic Mycobacterium avium complex lung disease. Chest. 2014;146:276-82. http://dx.doi.org/10.1378/ chest.13-2538

34. Jeong BH, Jeon K, Park HY, Kim SY, Lee KS, Huh HJ, et al. Intermittent antibiotic therapy for nodular bronchiectatic Mycobacterium avium complex lung disease. Am J Respir Crit Care Med. 2015;191:96-103. http://dx.doi.org/10.1164/ rccm.201408-15450C

Address for correspondence: Sarah K. Brode, Toronto Western Hospital, 7E-453, 399 Bathurst St, Toronto, ON M5T 2S8, Canada; email: sarah.brode@uhn.ca 